\title{
CW-Dye Laser Spectrometer for Ultrahigh Resolution Spectroscopy: Design and Performance
}

\author{
Hassan El-Kashef \\ Physics Department, Faculty of Science, Tanta University, Tanta, Egypt \\ Email: hassanelkashef51@yahoo.com
}

Received 28 June 2014; revised 21 July 2014; accepted 14 August 2014

Copyright (C) 2014 by author and Scientific Research Publishing Inc.

This work is licensed under the Creative Commons Attribution International License (CC BY). http://creativecommons.org/licenses/by/4.0/

\section{(c) 7 Open Access}

\section{Abstract}

This work is devoted to building-up of ultrahigh resolution cw-dye laser spectrometer system. This system used self-frequency-stabilized and temperature-compensated plano-confocal reference cavity. The one-way propagation is achieved using new construction of optical diode. The laser frequency selection and tuning is accomplished using Mach-Zehnder interferometer of free spectral range $42.5 \mathrm{GHz}$. In combination with computerized tunable radio frequency technique, this system is capable of a resolution of about $\pm 1 \mathrm{KHz}$. Applications for measuring high lying, weakly occupied metastable states of free atoms (line $548.792 \mathrm{~nm}$ of V-51) are investigated to a high degree of accuracy. The results of the constants $A$ and $B$ of the hfs as measured by fluorescence spectroscopy show that $A=160.762$ and $B=-\mathbf{1 7 . 9 1 8}$, while the obtained results for the hfs constants $A$ and $B$ as measured by laser-RF double resonance technique give $A=160.9950$ and $B=-17.3358$.

\section{Keywords}

Dye Laser, Optical Spectroscopy, Radio Frequency Technique, Hyperfine Structure of Atoms

\section{Introduction}

In the last years, since the development of dye lasers, the atomic hyperfine structure (hfs) particularly for the elements of not closed 3d-levels was precisely investigated. On the other hand, it is very important to test the agreement of the developed hfs theory with the experimental results [1] [2]. The desired hfs investigation precision can be achieved if high frequency transitions are detected using laser spectroscopic methods. One of these methods is the ABMR-LIRF (Atomic Beam Magnetic Resonance detected by Laser-Induced Resonance Fluorescence) [3]. S. Kroell et al. measured the hyperfine structure of ${ }^{51} \mathrm{~V}$ using Doppler free saturated absorption 
and polarization spectroscopy [4], different experimental investigations of the hyperfine structure of alkali atoms [5], atomic Cobalt spectrum [6], ${ }^{135,137} \mathrm{Ba}$ [7], and Rubidium [8]. This Work describes the design and performance of a stabilized dye laser spectrometer efficiently suitable for laser spectroscopic detection of high frequency transitions between hfs components of metastable states. The particular elements which are considered as candidates for such investigations are: Scandium, Titanium, Chromium, Manganese, Iron, Cobalt, Copper, Zinc, Iridium, Platinium, Gold, Thallium, Lead, Zirconium, Molybdenum, Ruthenium, Rhodium, Silver, Barium and others.

\section{Tunable Single Frequency Dye Laser}

The dye laser system consider as the central part of the spectrometer, to achieve high precision measurements of the hfs splitting of free atoms, Its design must fulfill the following characteristics: 1) Broadband continuous tunability of wavelength. This is important for investigation of the fine structure of each spectral line. 2) For guarantee enough selectivity of certain excited hfs component, the laser beam must has narrow linewidth (smaller than the natural linewidth of the atomic transitions). 3) For intensive excitation of many atoms and accordingly high detection sensitivity specially for weak transition probability, the dye laser must has high spectral power density. 4) Because of transition probability depends on the precise determination of the excited line, therefore the laser system must has high frequency stability. 5) The cw-dye laser must be capable to lasing in a wide range $(\approx 50 \mathrm{GHz})$ of single frequency scan. This is to orient the measurements of the HFS splitting with high resolution fluorescence spectroscopy. These characteristics are realized in the present development of high power and single frequency stabilized ring dye laser system.

The optical resonator geometry has two spherical mirrors $(\mathrm{R}=50 \mathrm{~mm})$, three plane mirrors and a MachZehnder interferometer inserted in the corner of the cavity instead of one mirror as shown in Figure 1.

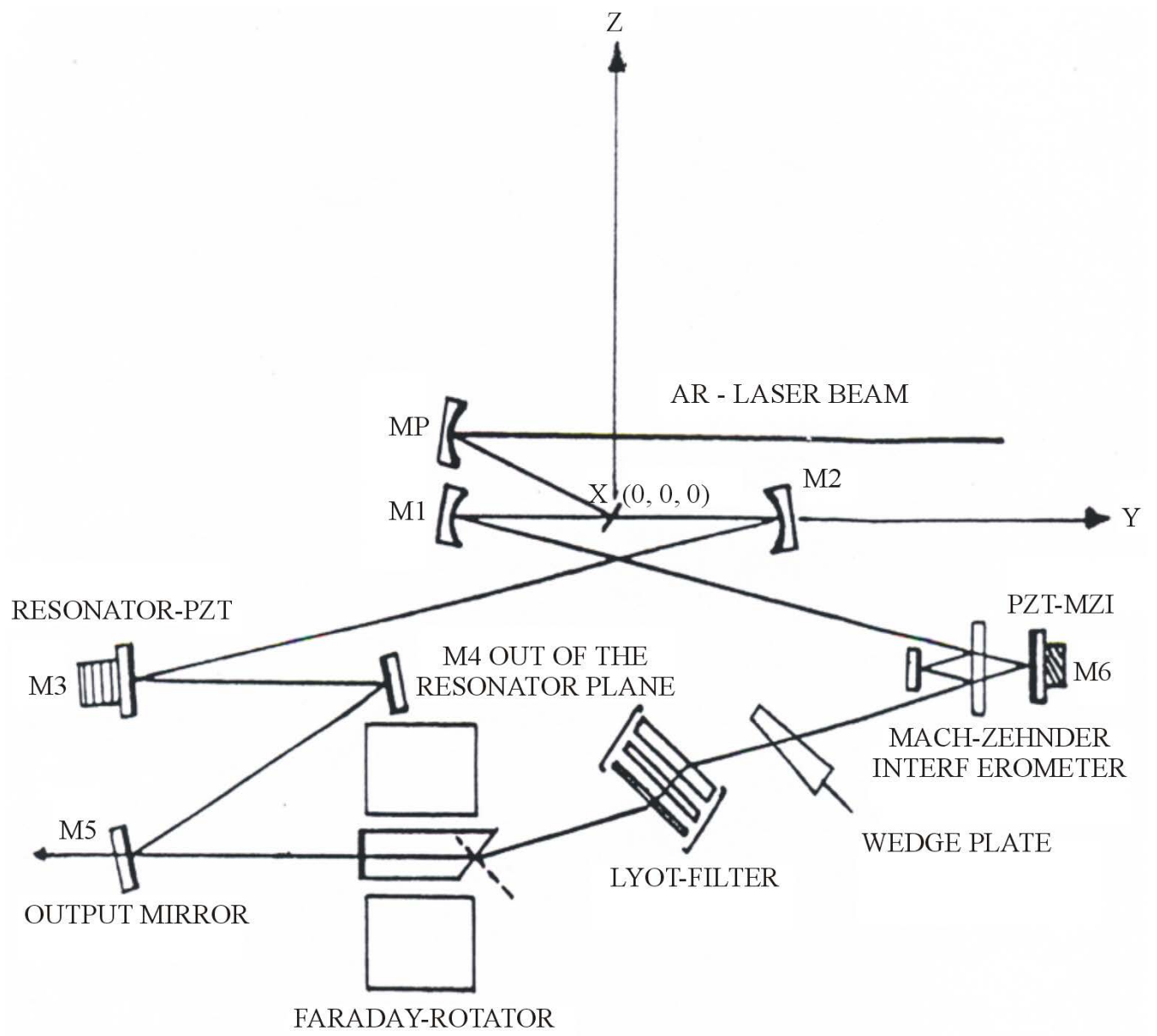

Figure 1. The ring dye laser system. 
The overall laser cavity circumference is about $\mathrm{L}=600 \mathrm{~mm}$. This is ensure a compact spectroscopic coherent light source (the cavity free spectral range-FSR $=500 \mathrm{MHz}$ ). One resonator arm has a tight focus and contains the dye jet stream, while the other parallel arm is used for insertion of the various wavelength selection elements. For obtaining high optical quality jet stream, a dye circulation system and jet nozzle is new constructed and optimized [9] [10]. The one-way propagation of a laser beam is achieved using a design form of an optical diode. It consists of two components: the first one consists of a system of three mirrors (M3-M4-M5) as in Figure 1. The arrangement of the system is constructed so that the mirror M4 lie out the resonator plane where its height is proportional to the polarization angle. More details about this arrangement are described in [11]. The second is a wedged Faraday rotator. A glass rod (BK-7, Firma Schott, Mainz, Germany) of $20 \mathrm{~mm}$ length is placed in cylinderical stack of high permenant magnetic field (9 kOs). The polarization rotation can be achieved by translational motion of the Faraday rotator perpendicular to the direction of the laser beam (Z-axis) as in Figure 1. The measured linewidth of the laser emission using this optical diode is $12^{\circ} \mathrm{A}$ without introducing of the frequency selective elements. Coarse tuning with a linewidth of $40 \mathrm{GHz}$ is accomplished with a three-parallel-plate quartz crystal filter of thickness ratio 1:4:16.

For obtaining high power laser frequency scanning, a Mach-Zehnder interferometer with FSR $=42.5 \mathrm{GHz}$ is designed and inserted in the corner of the cavity instead of one mirror as shown in Figure 1. It consists of two high reflector plane mirrors, and the beam splitter (thickness $=4.5 \mathrm{~mm}$ ), reflectivity $=50 \% \pm 5 \%$. The MZI produced optimum output power and single frequency scanning with a linewidth of $\pm 20 \mathrm{MHz}$ [12]. The MZI transition peak is controlled by the voltage output from a digital-to-analog converter amplified by high voltage ramp generator which applied to a pizo-mirror translator. Using such filter and MZI combination, a single frequency scan up to $50^{\circ} \mathrm{A}$ and dye laser mode hope of $\mathrm{c} / \mathrm{L}=500 \mathrm{MHz}$ can be achieved. Continuous frequency scanning requires synchronous tracking of the effective cavity length using a PZT translator with respect to the MZI spacing.

The dye laser spectrometer is optimized using the dye Rh $6 \mathrm{G}$ dissolved in ethylene glycol as active medium. It produces a single output power of $580 \mathrm{~mW}$ using $2 \mathrm{~W}$ pump power at $514.5 \mathrm{~nm}$ (argon ion laser = Spectra Physica). The argon laser beam is coupled into the cavity as shown in Figure 2. The calculated conversion efficiency $=29 \%$. In order to carry out the measurements of the hfs of V-51, the dye Rh 110 dissolved in ethylene glycol is used. The dye laser produced the same conversion efficiency as with Rh $6 \mathrm{G}$.

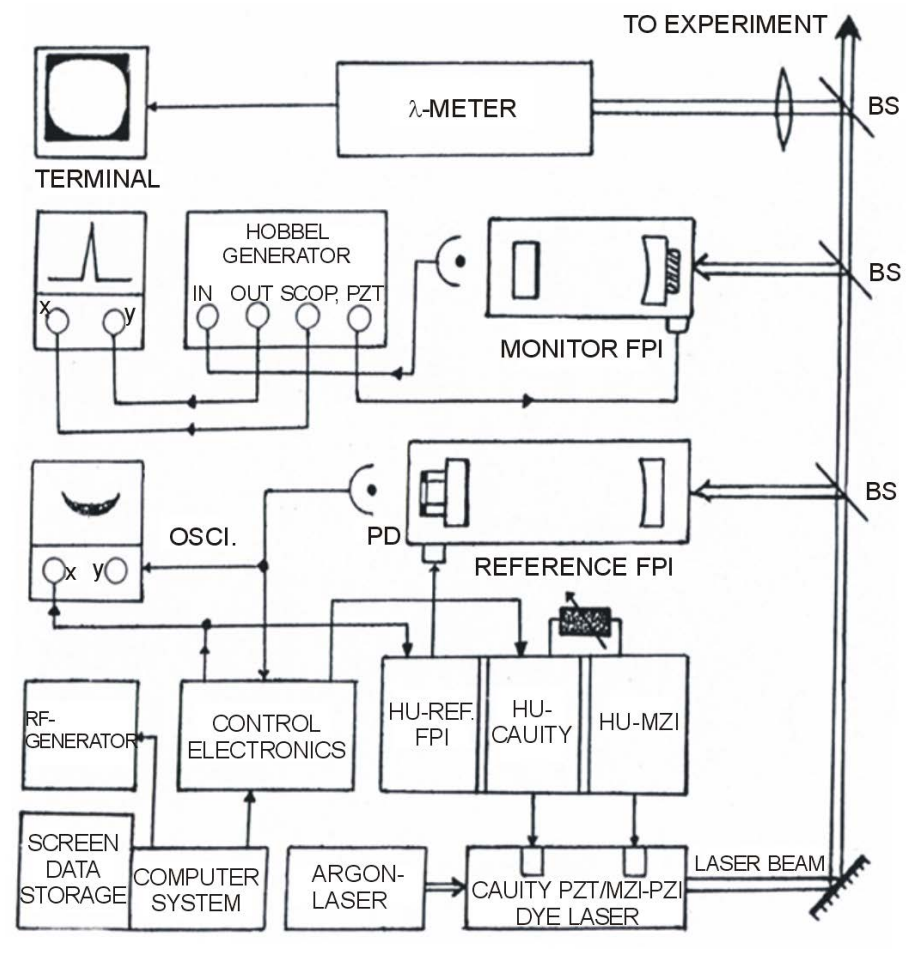

Figure 2. Schematic of computer-stabilized ring dye laser system. 
The dye laser wavelength is monitored using a $\lambda$-meter of Michelson type. An error in the absolute frequency determination of circa $10^{-7}$ is possible. This leads to frequency deviation of $\pm 50 \mathrm{MHz}$. A temperature compensated passively frequency stabilized plano-confocal optical spectrum analyzer (FSR $=2 \mathrm{GHz}$ ) is applied as a reference cavity. It used for calibration of the MZI and to provide frequency marker during scan. Several visible photodiode-operational amplifier detectors were used to monitor the power levels. The signals from these detectors were input to a multiplexed analog-to digital converter.

Errors arise from the output frequency noise or frequency jitter. This is mainly due to the noise generated in the flowing jet stream of dye solution. Accordingly, a servo electronic system is used to correct these laser frequency errors. This reduces the linewidth to something which is more useful for high resolution laser spectroscopy. An error signal is generated by tuning the reference cavity where the laser frequency sits on the side of its transmission peak. The laser intensity is also monitored with a photodetector and the two signals are subtracted so that a zero output occurs when the laser is tuned to the side of the transmission peak. The resulting error signal is fed back to resonator length control devices. These devices are just the wedge plate (wedge angle of 0.5 degree and $19 \mathrm{~mm}$ length) and the piezoelectricity-driven cavity mirror. In combination with radio frequency double resonance technique the effective linewidth of this laser system is reduced to the order of less than 10 $\mathrm{kHz}$. Laser frequency drift becomes that of reference cavity, or in this case less than $7.5 \mathrm{MHz}$ per hour. These systems will perform precision scans up to $50 \mathrm{GHz}$.

For automatic scanning of the dye laser an integrated element (computer) is used. Signals from the photodetectors of the laser and of the wavemeter are fed to a microcomputer via an interface electronics which convert the analog signals to digital. These signals are analyzed by the computer, and in combination with the operator commands, they are used to generate the appropriate signals for control the dye laser. These control signals are sent via interface to the electronic box. A schematic of a computer stabilized dye laser spectrometer shows Figure 2.

\section{Applications and Results}

To demonstrate the narrow linewidth and estimate the low drift rate of the present dye laser spectrometer, we have investigated the hyperfine structure of one previously unknown high lying weakly occupied metastable states in an V-51 atomic beam. The wavelength in air of this line is extracted from [13]:

$$
\left(3 \mathrm{~d}^{4} 4 \mathrm{~s}\right)^{2} \mathrm{H}_{11 / 2} \stackrel{548.792 \mathrm{~nm}}{\rightarrow} \rightarrow\left(3 \mathrm{~d}^{4} 4 \mathrm{P}\right)^{2} \mathrm{G}_{9 / 2}^{0}
$$

Figure 3 shows schematic diagram of the V-51 atomic beam apparatus for observation the hfs splitting components. It is evacuated to $2 \times 10^{-6}$ Torr using two oil diffusion pumps.

For the optical pumping and for finding the RF-transitions one needs a rough knowledge of the hfs splitting of the spectral lines. These information were obtained by conventional high resolution fluorescence spectroscopy using the present dye laser system. The wavelength of this laser is tuned to the mentioned green fine structure (fs) line. The frequency of the excited laser beam, which crossed rectangularly the well-collimated atomic beam was continuously tuned for the spectral line over the full range of its hfs. The resonance fluorescence represent the hfs splitting of the line is synchronously registered by a photomultiplier. Figure 4 shows the hyperfine splitting of the fine structure line $548.792 \mathrm{~nm}$. Figure 5 shows the spectrum of atomic transitions $\left({ }^{2} \mathrm{H}_{11 / 2} \rightarrow{ }^{2} \mathrm{G}_{9 / 2}^{0}\right)$. The inserted table gives the relative distances between the transitions of Figure 5 and its interpretation. Figure 6 shows a part of high resolution fluorescence spectrum of the investigated line $548.792 \mathrm{~nm}$ of V-51. The constants A and B of the hfs as measured by fluorescence spectroscopy gives $A=160.762$ and $B=-17.918$. A multiple pass system consists of two prisms is installed for improving the optical pumping efficiency. The design allows the laser beam to interact for at least 13 times with the atomic beam. As shown in Figure 3 the laser beam 1 was tuned to one of the hfs components, in order to deplete selectively one metastable hfs state of the lower hfs level. This causes an equivalent decrease of the resonance fluorescence signal in the second interaction region. In a RF-loop placed between the two interaction regions (Figure 3), transitions were induced between this depleted and its neighbouring hfs state, with the result of equalizing their populations. The RF resonance is detected with a photomultiplier by a strong increase of the laser induced fluorescence light at the second interaction region. During the measurements, the laser frequency is stabilized on one hfs component and the fluorescence light intensity registered as a function of the irradiated high frequency. The frequency of the chopped RF-field is produced either by an RF-generator type Rohde and Schwartz SLRD ( $v_{\mathrm{RF}}>1 \mathrm{GHz}$ ) or a magnetron 
$\left(v_{\mathrm{RF}}<1 \mathrm{GHz}\right)$, and tuned on-line by the computer system which simultaneously processed the RF resonance signal from the photomultiplier. The obtained results for the hfs constants A and B as measured by laser-RF double resonance technique give $\mathrm{A}=160.9950$ and $\mathrm{B}=-17.3358$.

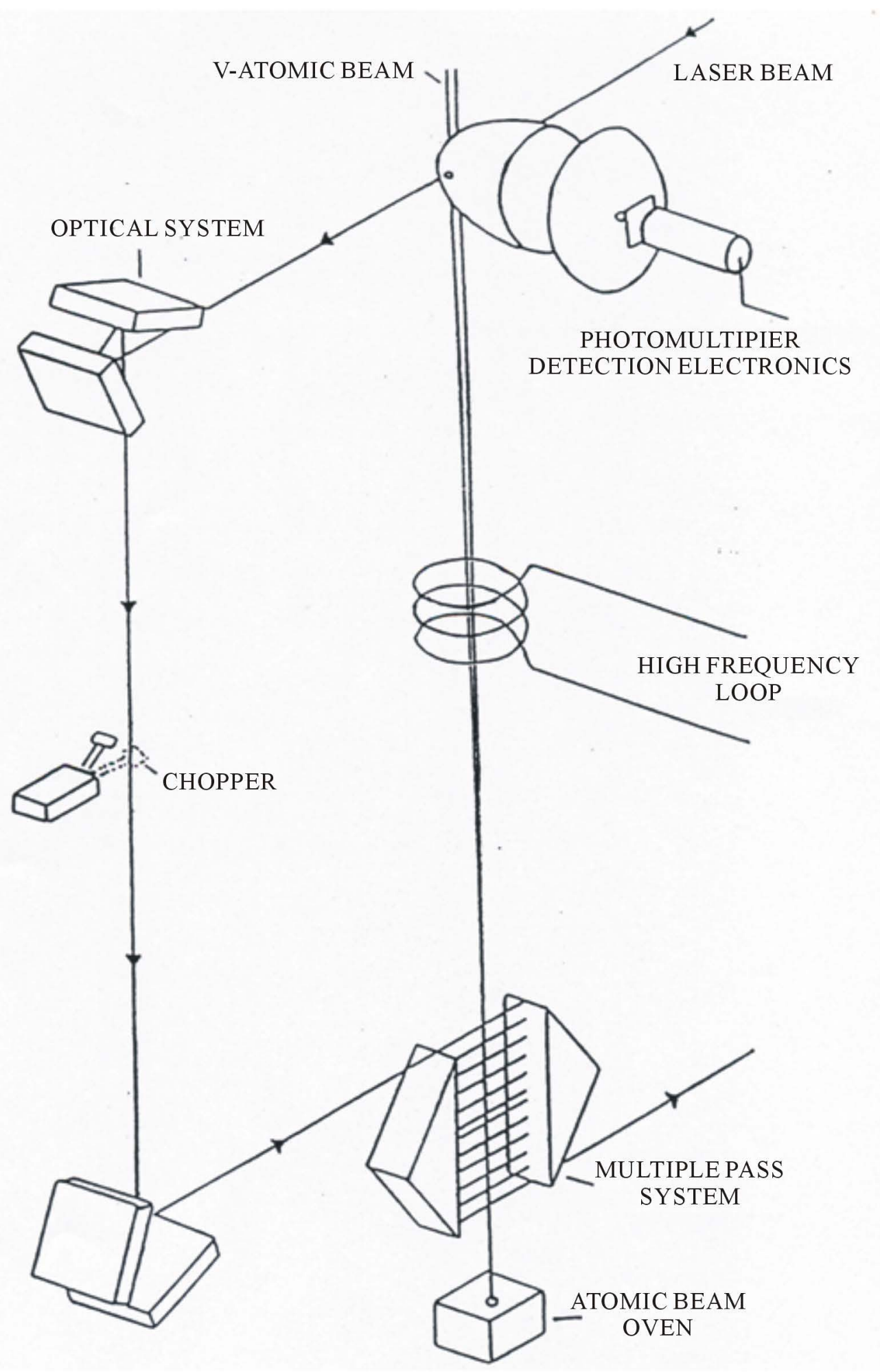

Figure 3. Schematic diagram of the V-51 atomic beam apparatus. 


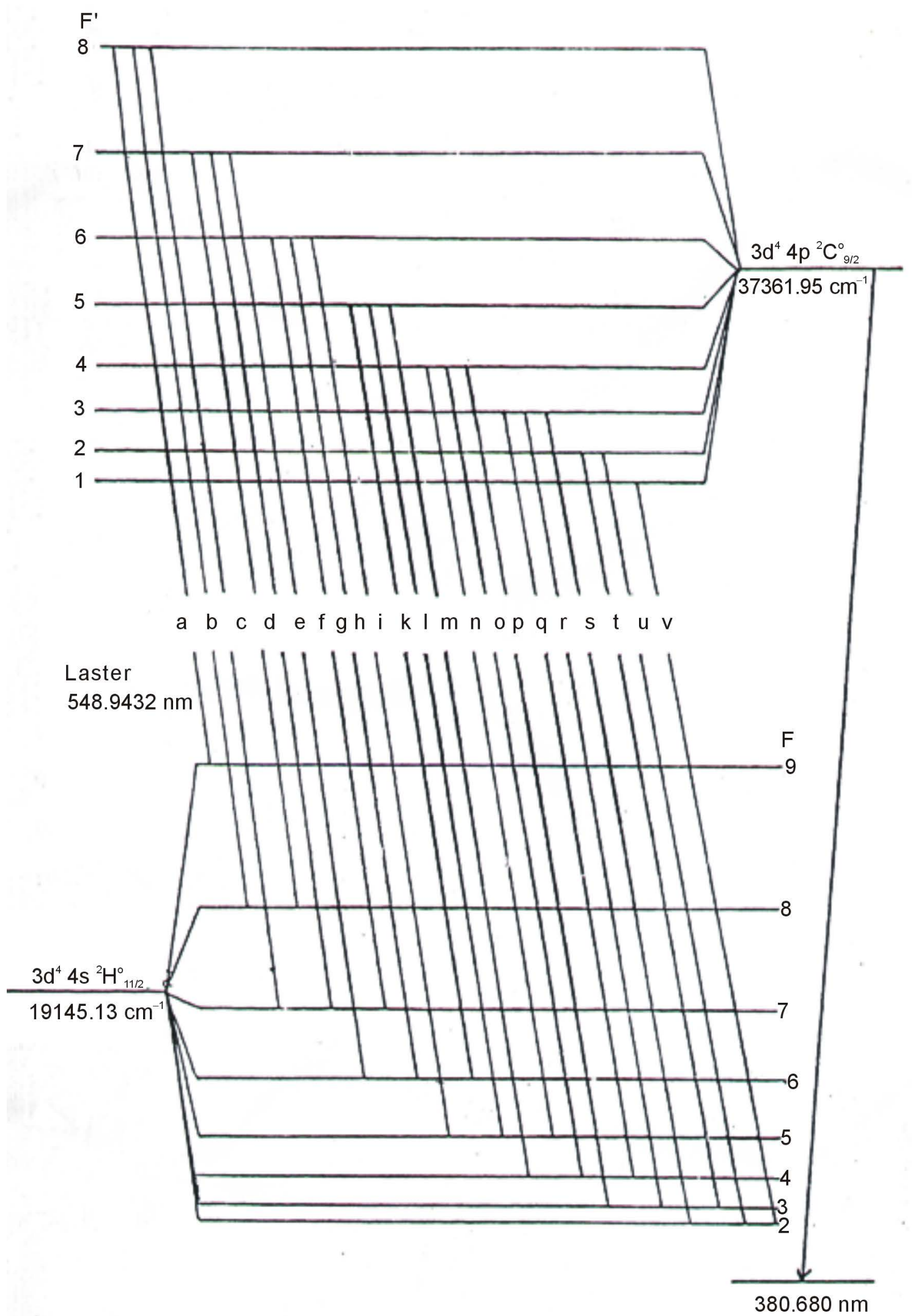

Figure 4. The hyperfine splitting of the fine structure line $548.792 \mathrm{~nm}$. 


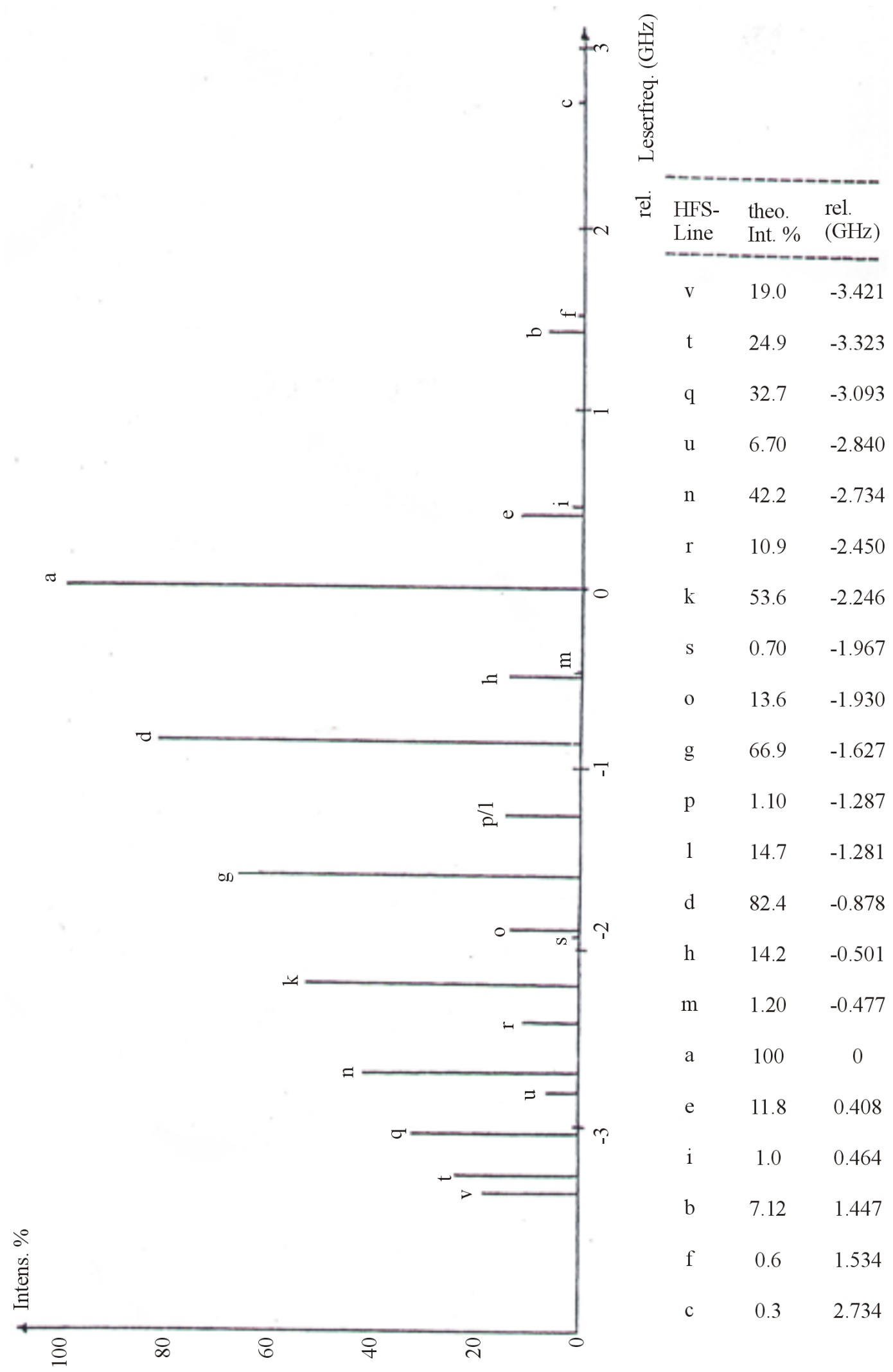

Figure 5. The spectrum of atomic transitions $\left({ }^{2} \mathrm{H}_{11 / 2} \rightarrow{ }^{2} \mathrm{G}_{9 / 2}^{0}\right)$. 


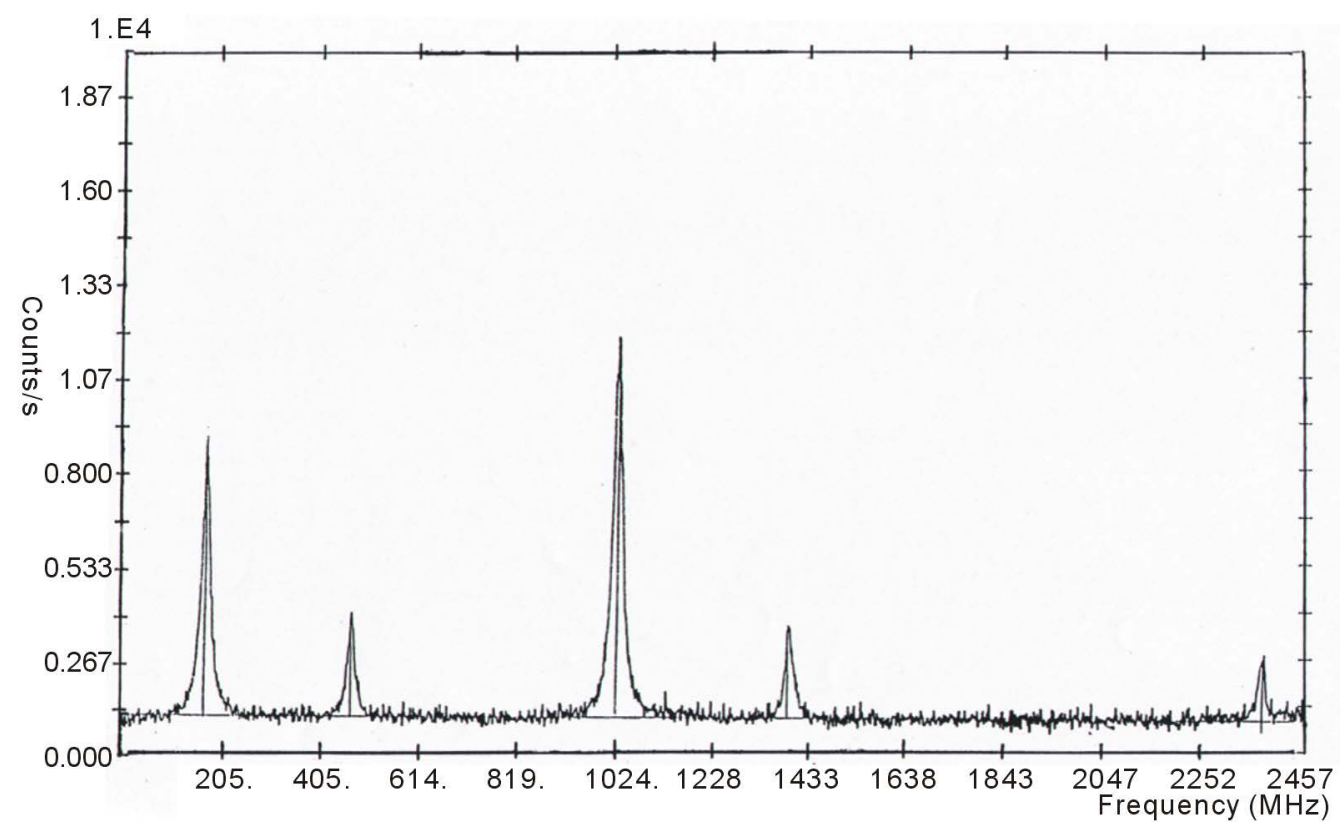

Figure 6. A part of high resolution fluorescence spectrum of the line $\lambda=548.796 \mathrm{~nm}$ of V-51.

\section{Conclusion}

A dye laser spectrometer with extremely high spectral resolution has been developed. The single mode operation is accomplished by using MZI without addition of more etalons in the cavity. The one-way propagation is achieved using a wedged Faraday rotator and an arrangement system of three mirrors. The laser frequency is stabilized to a suitable resonance frequency of PZT temperature-compensated plano-confocal Fabry-Perot Interferometer. By incorporating a computer into the ring laser system and a tunable radio frequency generator, a number of important features are obtained. Some of these features include stacking of scans, mode-hop detection, scan linearization, and automatic MZI peaking. This dye laser spectrometer has been used successfully for experimental investigation of the hfs of unknown high lying weakly occupied metastable states of V-51 and the hfs constants A and B are determined to a high degree of accuracy

\section{References}

[1] Childs, W.J. and Goodman, L.S. (1967) Hyperfine Structure of Nine Levels in Two Configurations of V ${ }^{51}$. I Experimental. Physical Review Letters, 156, 64-70. http://dx.doi.org/10.1103/PhysRev.156.64

[2] Childs, W.J. (1973) Fine and Hyperfine Structure of Free Atoms. Bonn University, Institute fuer Angevandte Physik, Germany

[3] Ertmer, W. and Hofer, B. (1976) Zero-Field Hyperfine Structure Measurements of the Metastable States $3 d^{2} 4 s^{4} F_{3 / 2,}$ 9/2 of ${ }^{45}$ Sc Using Laser Fluorescence Atomic-Beam-Magnetic-Resonance Technique. Zeitshrift fuer Physik A, 276, 9-14.

[4] Kroel, S. and Persson, A. (1985) Hyperfine Sstructure Measurements in ${ }^{51}$ V Using Doppler Free Saturated Absorption and Polarization Spectroscopy. Optics Communications, 54, 277-282. http://dx.doi.org/10.1016/0030-4018(85)90354-2

[5] Arimondo, E., Inguscio, M. and Violing, P. (1977) Experimental Determinations of the Hyperfine Structure in the Alkali Atoms. Review of Modern Physics, 49, 31-75. http://dx.doi.org/10.1103/RevModPhys.49.31

[6] Guthoehrleinnd, G.H. and Keller, H.P. 1990) Doppler-Free Laser Spectroscopic Investigations of the Hyperfine Structure in the Atomic Cobalt. Spectrum. Zeitschrift fuer Physik D—Atoms, Molecules and Clusters, 17, 181-193. http://dx.doi.org/10.1007/BF01437898

[7] Gustausson, M., Olsson, G. and Rosen, A. (1979) Hperfine Structure Investigations in the 6s5d Configuration of ${ }^{235} \mathrm{Ba}$ and ${ }^{137}$ Ba. Zeitschrift fuer Physik A-Atoms and Nucle, 290, 231-243. http://dx.doi.org/10.1007/BF01408538

[8] Kim, J.-T. Zhen, L., Kapitanov, V., Kim, H.S. Park, J.P. and Park, S.-H. (2011) Hyperfine Structure Measurement of Rubidium atom and Tunable Diode Laser Stabilization by Using Sagnac Interferometer. Journal of Nanoscience and Nanotechnology, 6, 3559-3561. http://dx.doi.org/10.1166/jnn.2006.056 
[9] El-Kashef, H. and Hassan G.E. (1992) Stability Conditions of the Dye-Laser Circulation System. Acta Physica Slovaca, 42, 305-309.

[10] El-Kashef, H. (1993) New High Interferometric Quality Dye Laser Jet Nozzle. Optics Communications, 100, $141-146$.

[11] Kriz, H. (1980) Aufbau eines Ein Moden Farbst off Ringlaser Diplomarbeit. Bonn University, Instutite fuer Angewandte Physik, Germany.

[12] El-Kashef, H. (1992) New Development of Mach-Zehnder Interferometer for Laser Frequency Selection and Tuning. Journal of Modern Optics, 39, 43-47. http://dx.doi.org/10.1080/713823357

[13] Sugar, J. and Corliss, C. (1978) Energy Levels of Vanadium, V I through V XXIII. Journal of Physical Chemistry Reference Data, 7, 1191. http://dx.doi.org/10.1063/1.555578 
Scientific Research Publishing (SCIRP) is one of the largest Open Access journal publishers. It is currently publishing more than 200 open access, online, peer-reviewed journals covering a wide range of academic disciplines. SCIRP serves the worldwide academic communities and contributes to the progress and application of science with its publication.

Other selected journals from SCIRP are listed as below. Submit your manuscript to us via either submit@scirp.org or Online Submission Portal.
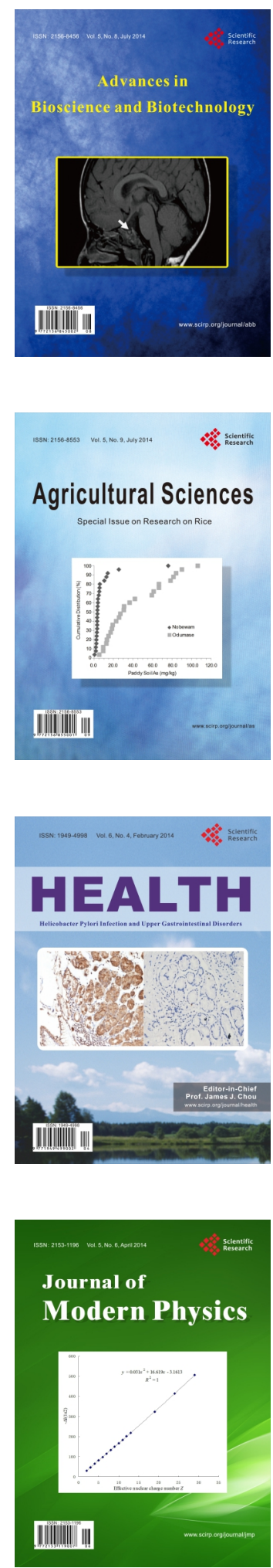
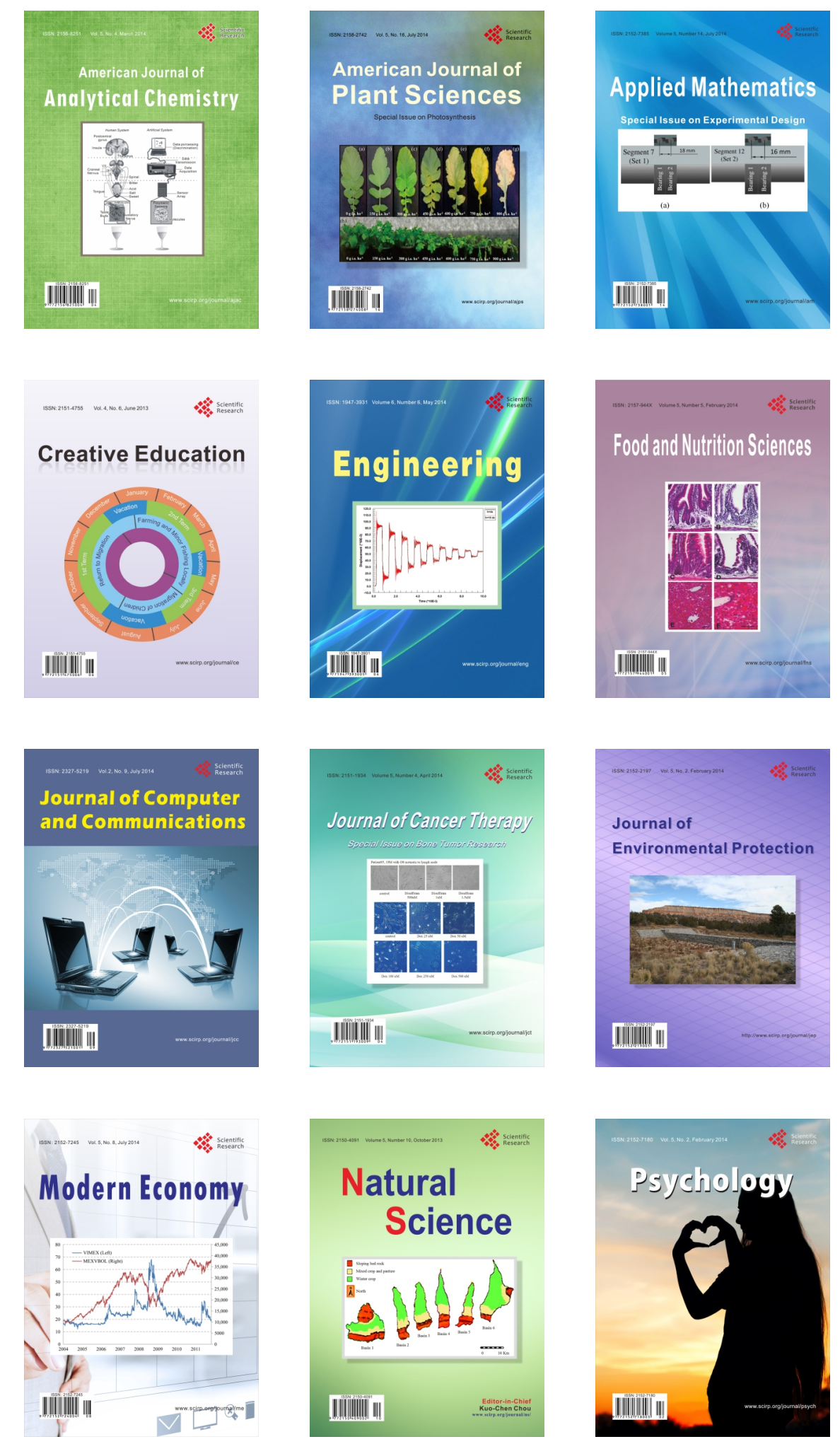